Bull. Austral. Math. Soc.

VOL. 66 (2002) [135-148]

\title{
ON FRÉCHET ALGEBRAS OF POWER SERIES
}

\author{
S.J. BhatT AND S.R. PATEL
}

If the indeterminate $X$ in a Fréchet algebra $A$ of power series is a power series generator for $A$, then either $A$ is the algebra of all formal power series or is the Beurling-Fréchet algebra on non-negative integers defined by a sequence of weights. Let the topology of $A$ be defined by a sequence of norms. Then $A$ is an inverse limit of a sequence of Banach algebras of power series if and only if each norm in the defining sequence satisfies certain closability condition and an equicontinuity condition due to Loy. A non-Banach uniform Fréchet algebra with a power series generator is a nuclear space. A number of examples are discussed; and a functional analytic description of the holomorphic function algebra on a simply connected planar domain is obtained.

\section{INTRODUCTION AND EXAMPLES}

A Fréchet algebra is a linear associative algebra $A$ with identity and having a Hausdorff topology defined by a sequence $\left(p_{k}\right)_{k \geqslant 1}$ (assumed increasing without loss of generality) of submultiplicative seminorms on it such that $A$ is complete in this topology. Such an $A$ is an inverse limit of a sequence of Banach algebras ([5]). A Fréchet algebra $A$ is called a uniform Fréchet algebra if for each $k \geqslant 1$ and for each $x \in A, p_{k}\left(x^{2}\right)=p_{k}(x)^{2}$. An element $x$ in a Fréchet algebra $A$ is a power series generator for $A$ if each $y \in A$ is of the form $y=\sum_{n=0}^{\infty} \lambda_{n} x^{n}, \lambda_{n}$ complex scalars, such that $\sum_{n=0}^{\infty}\left|\lambda_{n}\right| p_{k}\left(x^{n}\right)<\infty$ for all $k$. Let $\mathcal{F}$ be the Fréchet algebra of all formal power series $f=\sum_{n=0}^{\infty} \lambda_{n} X^{n}$ having complex coefficients $\lambda_{n}$ and in an indeterminate $X$ with the weak topology defined by the projections $\pi_{m}: \mathcal{F} \rightarrow \mathbb{C}, m \in \mathbb{Z}^{+}$, where $\pi_{m}(f)=\lambda_{m}$. A defining sequence of seminorms for $\mathcal{F}$ is $\left(p_{k}\right)$,

$$
p_{k}\left(\sum_{n=0}^{\infty} \lambda_{n} X^{n}\right)=\sum_{n=0}^{k}\left|\lambda_{n}\right| .
$$

\section{Received 18th February, 2002}

The first author is thankful to H.V. Dedania for a discussion. The second author gratefully acknowledges the support of the Council of Scientific and Industrial Research (Government of India) in the form of CSIR Research Fellowship. Both the authors are thankful to the referees for constructive suggestions.

Copyright Clearance Centre, Inc. Serial-fee code: 0004-9727/02 \$A2.00+0.00. 
A Fréchet algebra of power series is a subalgebra $A$ of $\mathcal{F}$ which is a Fréchet algebra containing $X$ such that the inclusion $A \hookrightarrow \mathcal{F}$ is continuous $([7,8])$. A weight function on $\mathbb{Z}^{+}$is a function $\omega: \mathbb{Z}^{+} \rightarrow \mathbb{R}$ such that for all $m, n \in \mathbb{Z}^{+}, \omega(m+n) \leqslant \omega(m) \omega(n)$ and $\omega(n)>0$. The present paper is concerned with the following two questions on Fréchet algebras of power series $A$.

(1) When is $X$ a power series generator for $A$ ?

(2) When is $A$ isomorphic to an inverse limit of Banach algebras of power series?

We show that $X$ is a power series generator for $A$ if and only if $A$ is either $\mathcal{F}$ or is the Beurling-Fréchet algebra

$$
\ell^{1}\left(\mathbb{Z}^{+}, \omega\right):=\bigcap_{k=1}^{\infty} \ell^{1}\left(\mathbb{Z}^{+}, \omega_{k}\right)
$$

defined by a sequence $\boldsymbol{\omega}=\left(\omega_{k}\right)$ of weight functions on $\mathbb{Z}^{+}$. It is also shown that if the topology of $A$ is defined by a sequence of norms $\left(p_{k}\right)$ then $A$ is an inverse limit of a sequence of Banach algebras of power series if and only if each $p_{k}$ is closable satisfying the condition

(E): For each $m \in \mathbb{Z}^{+}$, there exists $c_{m, k}>0$ such that

$$
\left|\pi_{m}(f)\right| \leqslant c_{m, k} p_{k}(f) \text { for all } f \in A \text {. }
$$

The existence of a $\left(p_{k}\right)$ satisfying $(\mathrm{E})$ is equivalent to Loy's condition (E) $([\mathbf{7}, \mathbf{8}])$; whereas closability of a norm on a normed algebra of power series is a necessary and sufficient condition for the completion to be a Banach algebra of power series. This implies the uniqueness of the Fréchet space topology and automatic continuity of derivations on the Beurling-Fréchet algebra $\ell^{1}\left(\mathbb{Z}^{+}, \boldsymbol{\omega}\right)$. We also show that a non-Banach uniform Fréchet algebra with a power series generator is necessarily nuclear as a locally convex space. The paper ends with functional analytical characterisations of certain holomorphic function algebras on planar domains.

Our definition of a Fréchet algebra with a power series generator is motivated by $\mathrm{Al}$ lan's definition of a Banach algebra with a power series generator ([1]). Banach algebras of power series have already been established as an important aspect of contemporary Banach algebra theory. Though Fréchet algebras of power series have been considered earlier by Loy $([7,8])$; recently they - and more generally, the power series ideas in general Fréchet algebras - have acquired significance in understanding the structure of a Fréchet algebra $([2,3])$. We end this introduction by giving few examples of Fréchet algebras of power series with the objective of fixing notation. 
EXAmple 1.1. The indeterminate $X$ is a power series generator for $\mathcal{F}$, since for any

$$
f=\sum_{n=0}^{\infty} \lambda_{n} X^{n}, \sum_{n=0}^{\infty}\left|\lambda_{n}\right| p_{k}\left(X^{n}\right)=\sum_{n=0}^{k}\left|\lambda_{n}\right|=p_{k}(f)
$$

EXAMPLE 1.2. The Beurling-Banach algebra

$$
\ell^{1}\left(\mathbb{Z}^{+}, \omega\right):=\left\{f=\sum_{n=0}^{\infty} \lambda_{n} X^{n} \in \mathcal{F}:|f|_{\omega}:=\sum_{n=0}^{\infty}\left|\lambda_{n}\right| \omega(n)<\infty\right\}
$$

is a Banach algebra of power series with norm $|\cdot|_{\omega}$ and having a power series generator $X$. Given an increasing sequence $\omega=\left(\omega_{k}\right)_{k \geqslant 1}$ of weight functions, the algebra

$$
\ell^{1}\left(\mathbb{Z}^{+}, \omega\right):=\bigcap_{k=1}^{\infty} \ell^{1}\left(\mathbb{Z}^{+}, \omega_{k}\right)
$$

is a Fréchet algebra with power series generator $X$, the topology being defined by $\left\{|\cdot|_{\omega_{k}}: k \in \mathbb{N}\right\}$.

EXAMPLE 1.3. Let

$$
D=\{z \in \mathbb{C}:|Z| \leqslant 1\}, \quad U=\{z \in \mathbb{C}:|Z|<1\}
$$

Let $A(D)$ be the disc algebra on $D$. Let $\omega=\left(\omega_{k}\right)_{k \geqslant 1}$ be as above, $\omega_{k}(n) \geqslant 1$ for all $k$, for all $n$. Let

$$
A\left(D, \omega_{k}\right):=\left\{f \in A(D): f(z)=\sum_{n=0}^{\infty} \frac{f^{(n)}(0)}{n !} z^{n},|f|_{\omega_{k}}:=\sum_{n=0}^{\infty} \frac{\left|f^{(n)}(0)\right|}{n !} \omega_{k}(n)<\infty\right\} .
$$

Then $\left(A\left(D, \omega_{k}\right),|\cdot|_{\omega_{k}}\right)$ is a Banach subalgebra of $A(D)$; and identifying $f$ with $\sum_{n=0}^{\infty}\left(f^{(n)}(0) / n !\right) X^{n}$, it is a Banach algebra of power series having power series generator $\stackrel{n=0}{X}$. Thus $A(D, \omega)=\bigcap_{k=1}^{\infty} A\left(D, \omega_{k}\right)$ is a power series generated Fréchet algebra of power series. Similarly, one can consider the algebras $H^{\infty}(U, \omega), H\left(U, \omega_{k}\right)$ and $H(U, \omega)$. Notice that both $A(D)$ and $H^{\infty}(U)$ are uniform Banach algebras of power series which are not power series generated.

EXAMPLE 1.4. Let $H(U)$ be the Fréchet algebra of all holomorphic functions on $U$ with the compact-open topology defined by the norms

$$
\|f\|_{\infty, r_{k}}:=\sup \left\{|f(z)|:|z| \leqslant r_{k}\right\},\left(r_{k}\right)_{k \geqslant 1}
$$


being an increasing sequence of positive numbers $r_{k}, 0<r_{k}<1$ and $r_{k} \rightarrow 1$. The radius of convergence of the Taylor series $f(z)=\sum_{n=0}^{\infty}\left(f^{(n)}(0) / n !\right) z^{n}$ of each $f \in H(U)$ is not less than 1 ; and

$$
\sum_{n=0}^{\infty} \frac{\left|f^{(n)}(0)\right|}{n !}\left\|z^{n}\right\|_{\infty, r_{k}} \leqslant \sum_{n=0}^{\infty} \frac{\left|f^{(n)}(0)\right|}{n !} r_{k}^{n}<\infty
$$

showing that $H(U)$ is a power series generated uniform Fréchet algebra of power series. It contains the Fréchet algebra $H(U, \omega):=\bigcap_{k=1}^{\infty} H\left(U, \omega_{k}\right)$. The Fréchet algebra $\mathbb{E}$ of entire functions with the compact-open topology defined by the norms $\left\{\|\cdot\|_{\infty, k}: k \in \mathbb{N}\right\}$ is a power series generated Fréchet algebra of power series, whose topology is also defined by $\left\{|\cdot|_{k}: k \in \mathbb{N}\right\}$,

$$
|f|_{k}=\sum_{n=0}^{\infty} \frac{\left|f^{(n)}(0)\right|}{n !} k^{n}
$$

EXAMPLE 1.5. Let $C^{k}(\Gamma)$ be the Banach algebra of all $C^{k}$-functions defined on the unit circle $\Gamma$. Let $A^{k}(\Gamma)=\left\{f \in C^{k}(\Gamma)\right.$ : the Fourier coefficients $\widehat{f}(n)=0$ for all $\left.n<0\right\}$. Identifying $f$ with its Fourier series, $A^{k}(\Gamma)$ is a Banach algebra of power series. Let

$$
A^{k+}(\Gamma)=\left\{f \in A^{k}(\Gamma):|f|_{k+}:=\sum_{n=0}^{\infty}|\widehat{f}(n)| n^{k}<\infty\right\}
$$

a power series generated Banach algebra with the norm $|\cdot|_{k+}$. Let

$$
\|f\|_{k}=\max _{0 \leqslant i \leqslant k}\left(\sup _{x \in \Gamma}\left|f^{(i)}(x)\right|\right) \text { for } f \in C^{\infty}(\Gamma)
$$

Then for any $k$, there exists $c>0$ such that $A^{k+1}(\Gamma) \subset A^{k+}(\Gamma) \subset A^{k}(\Gamma)$, and $\|f\|_{k} \leqslant|f|_{k+} \leqslant c\|f\|_{k+1}$ for all $f \in A^{k+1}(\Gamma)$. Thus

$$
A^{\infty}(\Gamma)=\bigcap_{k=0}^{\infty} A^{k}(\Gamma)=\bigcap_{k=0}^{\infty} A^{k+}(\Gamma)
$$

is a Fréchet algebra of power series having power series generator $e^{i \theta}$. It is a closed subalgebra of the Fréchet algebra $C^{\infty}(\Gamma)$. Like $\mathcal{F}$, and unlike any non-Banach uniform Fréchet algebra considered in Example 1.4 above, $A^{\infty}(\Gamma)$ is a $Q$-algebra in the sense that its invertible elements form an open set. The Gelfand space of $A^{\infty}(\Gamma)$ is $D$. 


\section{Power series generated Fréchet Algebras of POWER SERIES}

Theorem 2.1. Let $A$ be a Fréchet algebra of power series in an indeterminate $X$. Suppose that $X$ is a power series generator for $A$. Then $A$ is either $\mathcal{F}$ or the Beurling-Fréchet algebra $\ell^{1}\left(\mathbb{Z}^{+}, \omega\right)$ for an increasing sequence $\omega$ of weights on $\mathbb{Z}^{+}$.

An element $x$ in a Fréchet algebra $A$ generates a cyclic basis [6, Chapter III, Section 7] if each $f \in A$ can be uniquely expressed as $f=\sum_{n=0}^{\infty} \lambda_{n} x^{n}, \lambda_{n}$ being scalars. A seminorm $p$ on a Fréchet algebra $A$ having a power series generator $x$ is a power series generator seminorm if

$$
p\left(\sum_{n=0}^{\infty} \lambda_{n} x^{n}\right)=\sum_{n=0}^{\infty}\left|\lambda_{n}\right| p\left(x^{n}\right)
$$

for all $f=\sum_{n=0}^{\infty} \lambda_{n} x^{n} \in A$.

Lemma 2.2. Let $A$ be a singly generated Fréchet algebra with a cyclic basis generated by $x$. Then there exists a dense Fréchet subalgebra $A_{1}$ of $A$ such that

(i) $A_{1}$ is continuously embedded in $A$;

(ii) $A_{1}$ is a Fréchet algebra having a power series generator $x$; and

(iii) $A_{1}$ is a Banach algebra provided $A$ is a Banach algebra.

Proof: Let $\left(p_{k}\right)$ be an increasing sequence of seminorms defining the topology $\tau$ of $A$. Since each $f \in A$ has a unique expression $f=\sum_{n=0}^{\infty} \lambda_{n} x^{n}$, the extended real number

$$
q_{k}(f)=\sum_{n=0}^{\infty}\left|\lambda_{n}\right| p_{k}\left(x^{n}\right) \geqslant p_{k}(f)
$$

is well defined for each $k \in \mathbb{N}$. Let

$$
A_{1}=\left\{f \in A: q_{k}(f)<\infty \text { for all } k \in \mathbb{N}\right\}
$$

Given

$$
f=\sum_{n=0}^{\infty} \lambda_{n} x^{n}, g=\sum_{n=0}^{\infty} \mu_{n} x^{n}
$$

the absolute convergence of these series in $A$ implies that

$$
f+g=\sum_{n=0}^{\infty}\left(\lambda_{n}+\mu_{n}\right) x^{n}, f g=\sum_{n=0}^{\infty}\left(\sum_{m=0}^{n} \lambda_{m} \mu_{n-m}\right) x^{n}
$$


and for each $k$,

$$
\begin{aligned}
q_{k}(f+g) & \leqslant \sum_{n=0}^{\infty}\left|\lambda_{n}+\mu_{n}\right| p_{k}\left(x^{n}\right) \leq q_{k}(f)+q_{k}(g)<\infty, \\
q_{k}(f g) \leqslant \sum_{n=0}^{\infty} \sum_{m=0}^{n}\left|\lambda_{m}\right|\left|\mu_{n-m}\right| p_{k}\left(x^{m+n-m}\right) & \leqslant \sum_{n=0}^{\infty} \sum_{m=0}^{n}\left|\lambda_{m}\right|\left|\mu_{n-m}\right| p_{k}\left(x^{m}\right) p_{k}\left(x^{n-m}\right) \\
& \leqslant q_{k}(f) q_{k}(g)<\infty .
\end{aligned}
$$

This shows that $A_{1}$ is a subalgebra of $A$ and $\left(q_{k}\right)$ is an increasing sequence of submultiplicative seminorms on $A_{1}$ defining a topology $\tau_{1}$ finer than the relative topology $\tau$. We show that $\left(A_{1}, \tau_{1}\right)$ is complete.

Let $\left(y_{m}\right), y_{m}=\sum_{n=0}^{\infty} \lambda_{n, m} x^{n}$, be a $\tau_{1}$-Cauchy sequence in $A_{1}$. Then it is $\tau$ Cauchy, and $y_{m} \rightarrow y$ in $(A, \tau)$. Since $\phi_{m}(n)=\lambda_{n, m} x^{n}$ is $\tau$-Cauchy (in fact, uniformly $\tau$-Cauchy over $m$ ), there are scalars $\lambda_{n}$ such that $\phi_{m}(n) \rightarrow \lambda_{n} x^{n}$ in $\tau$ uniformly over $m$. Then for each $i$,

$$
\sum_{n=0}^{\infty}\left|\lambda_{n}\right| p_{i}\left(x^{n}\right)=\sum_{n=0}^{\infty} \lim _{m}\left|\lambda_{n, m}\right| p_{i}\left(x^{n}\right) \leqslant \underline{\lim }_{m} \sum_{n=0}^{\infty}\left|\lambda_{n, m}\right| p_{i}\left(x^{n}\right) \leqslant \underline{\lim }_{m} q_{i}\left(y_{m}\right)<\infty
$$

as $\left(y_{m}\right)$ is $\tau_{1}$-Cauchy. Further $\sum_{n=0}^{\infty} \lambda_{n} x^{n}$ converges in $(A, \tau) ;$ since for each $i$ and for each $m \geqslant n$,

$$
p_{i}\left(\sum_{k=0}^{m} \lambda_{k} x^{k}-\sum_{k=0}^{n} \lambda_{k} x^{k}\right)=p_{i}\left(\sum_{k=n+1}^{m} \lambda_{k} x^{k}\right) \leqslant \sum_{k=n+1}^{m}\left|\lambda_{k}\right| p_{i}\left(x^{k}\right) \rightarrow 0 \text { as } n, m \rightarrow \infty .
$$

Then we have $\sum_{n=0}^{\infty} \lambda_{n} x^{n}=y$ and $y_{m} \rightarrow y$ in $\left(A_{1}, \tau_{1}\right)$. Indeed for each $i$,

$$
\begin{aligned}
q_{i}\left(\sum_{n=0}^{\infty} \lambda_{n} x^{n}-y_{m}\right) & =\sum_{n=0}^{\infty}\left|\lambda_{n}-\lambda_{n, m}\right| p_{i}\left(x^{n}\right)=\sum_{n=0}^{\infty} \lim _{k}\left|\lambda_{n, k}-\lambda_{n, m}\right| p_{i}\left(x^{n}\right) \\
& \leqslant \underline{\lim }_{k} \sum_{n=0}^{\infty}\left|\lambda_{n, k}-\lambda_{n, m}\right| p_{i}\left(x^{n}\right)=\underline{\lim }_{k} q_{i}\left(y_{k}-y_{m}\right)
\end{aligned}
$$

showing that $\lim y_{m}=y=\sum_{n=0}^{\infty} \lambda_{n} x^{n}$ in $\left(A_{1}, \tau_{1}\right)$.

Corollary 2.3. Let $A$ be a Fréchet algebra having a power series generator $x$. Then $x$ generates a cyclic basis if and only if the topology of $A$ is defined by a sequence of power series generator seminorms.

ProOF OF THEOREM 2.1: By the uniqueness of the formal power series expression and the fact that $X$ is a power series generator for $A$, it follows that $\left\{X^{n}: n \in \mathbb{Z}^{+}\right\}$ 
is a cyclic basis for $A$. By Corollary 2.3, the Fréchet topology $\tau$ of $A$ is defined by an increasing sequence $\left(p_{n}\right)$ of power series generator seminorms; and for each $f=\sum_{n=0}^{\infty} \lambda_{n} X^{n}$ in $A$, we have $f=\lim _{n} \sum_{k=0}^{n} \lambda_{k} X^{k}$ in the topology $\tau$. For each $k$, $\omega_{k}: \mathbb{Z}^{+} \rightarrow[0, \infty), \omega_{k}(n)=p_{k}\left(x^{n}\right)$ define a separating sequence $\omega=\left(\omega_{k}\right)$ of functions satisfying $\omega_{k}(n+m) \leqslant \omega_{k}(n) \omega_{k}(m), \omega_{k}(n) \leqslant \omega_{k+1}(n)$ for all $k \in \mathbb{N}$ all $n, m \in \mathbb{Z}^{+}$. Let

$$
\ell^{1}\left(\mathbb{Z}^{+}, \omega\right):=\left\{f=\sum_{n=0}^{\infty} \lambda_{n} X^{n} \in \mathcal{F}: \sum_{n=0}^{\infty}\left|\lambda_{n}\right| \omega_{k}(n)<\infty \text { for all } k\right\} .
$$

Since each $p_{n}$ is a power series generator seminorm, $A \subset \ell^{1}\left(\mathbb{Z}^{+}, \omega\right)$. In fact, $A$ $=\ell^{1}\left(\mathbb{Z}^{+}, \boldsymbol{\omega}\right)$. Let $f=\sum_{n=0}^{\infty} \lambda_{n} X^{n} \in \ell^{1}\left(\mathbb{Z}^{+}, \boldsymbol{\omega}\right)$. Let $f_{n}=\sum_{k=0}^{n} \lambda_{k} X^{k}$. Since $X \in A$, each $f_{n} \in A$; and $\left(f_{n}\right)$ is a Cauchy sequence in $A$. Thus $f \in A$.

Now we are left with the following possibilities:

(a) All $\omega_{k}$ are weights.

(b) No $\omega_{k}$ is a weight.

(c) At least one $\omega_{k_{0}}$ fails to be a weight.

In the case (c), let $G=\left\{\omega_{k}: \omega_{k}\right.$ is not a weight $\}$. If $G$ is finite the corresponding $p_{k}$ may be deleted, if $G$ is infinite the corresponding $p_{k}$ can be taken to define the topology so reducing consideration to the case (b). Assume (b). Then for each $k$, there is $n$ such that $\omega_{k}(n)=0$. Then for all $m \in \mathbb{Z}^{+}, \omega_{k}(n+m) \leqslant \omega_{k}(n) \omega_{k}(m)=0$. It follows that for any $f \in \mathcal{F}$,

$$
f=\sum_{n=0}^{\infty} \lambda_{n} X^{n}, \sum_{n=0}^{\infty}\left|\lambda_{n}\right| \omega_{k}(n)<\infty
$$

for all $k$. Thus $A=\mathcal{F}$. This completes the proof.

REMARK 2.4. Let $(A,\|\cdot\|)$ be a normed algebra with a power series generator $x$. Let $A^{\sim}$ be the completion of $A$. Then $A^{\sim}$ need not be a Banach algebra having a power series generator $x$. Let $D=\{z \in \mathbb{C}:|z| \leqslant 1\}$. Let $A(D)$ be the disc algebra consisting of functions continuous on $D$ and holomorphic in the interior of $D$. It is a uniform Banach algebra with the supremum norm

$$
\|f\|_{\infty}=\sup \{|f(z)|: z \in D\} \text {. }
$$

Let $A$ be the subalgebra of $A(D)$ consisting of all functions $f$ whose Taylor series $f(z)=\sum_{n=0}^{\infty}\left(f^{(n)}(0) / n !\right) z^{n}$ is absolutely convergent on the circle $\Gamma$. For any $f \in A$,

$$
\sum_{n=0}^{\infty} \frac{\left|f^{(n)}(0)\right|}{n !}\left\|z^{n}\right\|_{\infty} \leqslant \sum_{n=0}^{\infty} \frac{\left|f^{(n)}(0)\right|}{n !}\|z\|_{\infty}^{n} \leqslant \sum_{n=0}^{\infty} \frac{\left|f^{(n)}(0)\right|}{n !}<\infty .
$$


This shows that $z$ is a power series generator for the normed algebra $\left(A,\|\cdot\|_{\infty}\right)$. Since $A$ contains polynomials in $z$, it follows from the Mergelyan Theorem that $A$ is dense in $A(D)$ which is identified with the completion of $A$. However, $z$ is not a power series generator for $A(D)$. Indeed, suppose that $z$ is a power series generator for $A(D)$. Then for any $f \in A(D)$, the Taylor series of $f$ is absolutely convergent on $D$. Also, since $A(D)$ is semisimple, the complete norms

$$
\|f\|_{\infty}=\sup \{|f(z)|: z \in D\}
$$

and

$$
\|f\|=\sum_{n=0}^{\infty} \frac{\left|f^{(n)}(0)\right|}{n !}\left\|z^{n}\right\|_{\infty}
$$

are equivalent. Thus for some $M>0,\|f\| \leqslant M\|f\|_{\infty}$ for all $f \in A(D)$. Hence, if $s_{m}(f)$ is the $m^{\text {th }}$ partial sum of the Taylor series of $f$, then

$$
\left\|s_{m}(f)\right\|_{\infty} \leqslant \sum_{n=0}^{m} \frac{\left|f^{(n)}(0)\right|}{n !}\left\|z^{n}\right\|_{\infty} \leqslant \sum_{n=0}^{\infty} \frac{\left|f^{(n)}(0)\right|}{n !}\left\|z^{n}\right\|_{\infty}=\|f\| \leqslant M\|f\|_{\infty}
$$

for all $m$ and all $f \in A(D)$. This contradicts a classical result that there exists a $f \in A(D)$ with $\left\|s_{m}(f)\right\|_{\infty} \geqslant\left|s_{m}(f)(1)\right|=O(\log m)$ (see [9, p. 264]). Note that $A(D)$ is a Banach algebra of power series which is not a power series generated Banach algebra.

Proposition 2.5. Let $(A,\|\cdot\|)$ be a normed algebra with a power series generator $x$. Let $A^{\sim}$ be the completion of $A$. Then $x$ is a power series generator for $A^{\sim}$ if there exists a power series generator norm $|\cdot|$ on $A$ equivalent to $\|\cdot\|$.

Proof: Given $y=\sum_{n=0}^{\infty} \lambda_{n} x^{n}$ in $A$, let $|\cdot|$ be a power series generator norm on $A$ equivalent to $\|\cdot\|$. Then $|y|=\sum_{n=0}^{\infty}\left|\lambda_{n}\right|\left|x^{n}\right|$. Now let $y \in A^{\sim}$. Choose a sequence

$$
y_{k}=\sum_{n=0}^{\infty} \lambda_{k, n} x^{n},\left|y_{k}\right|=\sum_{n=0}^{\infty}\left|\lambda_{k, n}\right|\left|x^{n}\right|
$$

such that $\left|y_{k}-y\right| \rightarrow 0$. Now

$$
\sup _{n \in \mathbb{Z}^{+}}\left|\lambda_{k, n}-\lambda_{l, n}\right|\left|x^{n}\right| \leqslant \sum_{n=0}^{\infty}\left|\lambda_{k, n}-\lambda_{l, n}\right|\left|x^{n}\right|=\left|y_{k}-y_{l}\right| \rightarrow 0
$$

shows that there exists a scalar sequence $\left(\lambda_{n}\right)_{n \in \mathbb{Z}^{+}}$such that $\lambda_{k, n} x^{n} \rightarrow \lambda_{n} x^{n}$ uniformly over $n$. Then

$$
\sum_{n=0}^{\infty}\left|\lambda_{n}\right|\left|x^{n}\right|=\sum_{n=0}^{\infty} \lim _{k}\left|\lambda_{k, n}\right|\left|x^{n}\right| \leqslant \underline{\lim }_{k} \sum_{n=0}^{\infty}\left|\lambda_{k, n}\right|\left|x^{n}\right|=\underline{\lim }_{k}\left|y_{k}\right|<\infty
$$


showing that $\sum_{n=0}^{\infty} \lambda_{n} x^{n} \in A^{\sim}$ and $\left|y_{k}-\sum_{n=0}^{\infty} \lambda_{n} x^{n}\right| \rightarrow 0$. Hence $y=\sum_{n=0}^{\infty} \lambda_{n} x^{n}$. This completes the proof.

\section{ARENS-Michael Decomposition}

Let us recall the Arens-Michael decomposition defined by a sequence $\left(p_{k}\right)$ of submultiplicative seminorms defining the topology $\tau$ of a Fréchet algebra $A$. We take $p_{1} \leqslant p_{2} \leqslant p_{3} \leqslant \ldots$. For each $k$, let $N_{k}=\left\{x \in A: p_{k}(x)=0\right\}$ an ideal. Let $A_{k}$ be the completion of $A / N_{k}$ in the norm $\left\|x+N_{k}\right\|_{k}=p_{k}(x)$. Then $\pi_{k}\left(x+N_{k+1}\right)=x+N_{k}(x \in A)$ extends as a norm decreasing surjective homomorphism $\pi_{k}: A_{k+1} \rightarrow A_{k}$ such that

$$
A_{1} \stackrel{\pi_{1}}{\longleftarrow} A_{2} \stackrel{\pi_{2}}{\longleftarrow} A_{3} \longleftarrow \ldots \leftarrow A_{k} \stackrel{\pi_{k}}{\longleftarrow} A_{k+1} \longleftarrow \ldots
$$

is an inverse limit sequence of Banach algebras; and bicontinuously $A=\lim _{k} A_{k}$. This is called the Arens-Michael decomposition of $A$ ([5]). This is the basic tool for studying Fréchet algebras.

Let $A$ be a Fréchet algebra of power series. Let $\tau$ denote the topology of coordinatewise convergence of $\mathcal{F}$. We call a seminorm $p$ on $A$ closable if for any $p$-Cauchy sequence $\left(f_{k}\right)$ in $A, f_{k} \rightarrow 0$ in $\tau$ implies that $p\left(f_{k}\right) \rightarrow 0$. We define $p$ to be of type (E) if given $m \in \mathbb{Z}^{+}$, there exists $c_{m}>0$ such that $\left|\pi_{m}(f)\right| \leqslant c_{m} p(f)$ for all $f \in A$ $([7,8])$. A seminorm of type $(E)$ is a norm.

Proposition 3.1. Let $A$ be a Fréchet algebra of power series. Let $p$ be a continuous submultiplicative seminorm on $A$. Let $\operatorname{ker} p=\{f \in A: p(f)=0\}$. Let $A_{p}$ be the completion of $A / \operatorname{ker} p$ in the norm $\|f+\operatorname{ker} p\|_{p}=p(f)$. Then the following are equivalent.

(i) $p$ is a norm and $A_{p}$ is a Banach algebra of power series.

(ii) $p$ is closable and of type (E).

Proof: (ii) implies (i). Since $p$ is of type (E), $p$ is a norm and each $\pi_{m}$ is $p$-continuous. By continuity, the inclusion $(A, p) \hookrightarrow \mathcal{F}$ extends to a continuous homomorphism $\phi: A_{p} \rightarrow \mathcal{F}$. Let $f \in \operatorname{ker} \phi$. Choose a sequence $\left(f_{n}\right)$ in $A$ such that $p\left(f_{n}-f\right) \rightarrow 0$. Hence $\left(f_{n}\right)$ is $p$-Cauchy. By the continuity of $\phi$, $\phi\left(f_{n}\right)=f_{n} \rightarrow \phi(f)=0$. Then $f_{n} \rightarrow 0$ in $\mathcal{F}$. By the closability, $p\left(f_{n}\right) \rightarrow 0$. Thus $f=0$ and $A_{p}$ is a subalgebra of $\mathcal{F}$. Thus $A_{p}$ is a Banach algebra of power series.

(i) implies (ii). That $A_{p}$ is a Banach algebra of power series implies that for each $m \in \mathbb{Z}^{+}$, there exists $c_{m}>0$ such that for all $f \in A,\left|\pi_{m}(f)\right| \leqslant c_{m} p(f)$. Also, let $\left(f_{n}\right) \subset A$ be such that $f_{n} \rightarrow 0$ in $(\mathcal{F}, \tau)$ and $p\left(f_{n}-f_{m}\right) \rightarrow 0$. Then for some $f \in A_{p}$, 
$p\left(f_{n}-f\right) \rightarrow 0$; and so $f_{n} \rightarrow f$ in $(\mathcal{F}, \tau), A_{p}$ being a Banach algebra of power series. Thus $f=0$; and (ii) follows.

Corollary 3.2. Let $A=\lim _{k} A_{k}$ be the Arens-Michael decomposition of a Fréchet algebra of power series $A$. Assume that each $p_{k}$ is a norm. Then each $A_{k}$ is a Banach algebra of power series if and only if each $p_{k}$ is a closable and of type (E).

Corollary 3.3. Let $A$ be a subalgebra of $\mathcal{F}$ containing $X$ which is a Banach algebra with a norm $\|\cdot\|$. Then $A$ is a Banach algebra of power series if and only if $\|\cdot\|$ is closable and of type $(\mathrm{E})$.

It is readily seen that a Fréchet algebra of power series $A$ satisfies Loy's condition (E) in [8] (that is, for some $c_{m}>0,\left(c_{m}^{-1} \pi_{m}\right)$ is equicontinuous) if and only if $A$ admits a continuous norms of type (E) if and only if the topology of $A$ is defined by a sequence of seminorms of type (E). Hence Corollary 3.2 gives the following from $[7,8]$. Note that a Beurling-Fréchet algebra $\ell^{1}\left(\mathbb{Z}^{+}, \omega\right)$ defined by a sequence of weights is expressible as an inverse limit of a sequence of Banach algebras of power series.

Corollary 3.4. Let $A$ be the Beurling-Fréchet algebra $\ell^{1}\left(\mathbb{Z}^{+}, \omega\right)$ defined by a sequence $\boldsymbol{\omega}$ of weights on $\mathbb{Z}^{+}$. The following hold.

(1) A has a unique Fréchet space topology as a topological algebra.

(2) Every derivation on $A$ is continuous

(3) A surjective homomorphism $\phi: B \rightarrow A$ from a Fréchet algebra $B$ is continuous.

It is instructive to exhibit the Arens-Michael decomposition of $\mathcal{F}$ defined by $\left(p_{k}\right)$, $p_{k}\left(\sum_{n=0}^{\infty} \lambda_{n} X^{n}\right)=\sum_{n=0}^{k}\left|\lambda_{n}\right|$. One has $N_{k}=X^{k+1} \mathcal{F} ;$ and $A_{k} \cong \ell^{1}\left(\mathbb{Z}_{(k)}^{+}\right)$, the convolution algebra on the finite semigroup $\mathbb{Z}_{(k)}^{+}=\{0,1,2, \ldots, k-1\}$ with addition modulo $k$. Certainly, it is not a subalgebra of $\mathcal{F}=\lim _{k} \ell^{1}\left(\mathbb{Z}_{(k)}^{+}\right)$. The role of closability, in Proposition 3.1 , is revealed by the following, whose proof we omit.

LEMMA 3.5. Let $(A,\|\cdot\|)$ be a normed algebra of power series continuously embedded in $\mathcal{F}$. Then the completion $A^{\sim}$ is a Banach algebra of power series if and only if $\|\cdot\|$ is closable.

Being an inverse limit of finite dimensional algebras, $\mathcal{F}$ is a nuclear Fréchet space. The following gives another class of such algebras. It also exhibits a significant difference between Banach algebras and Fréchet algebras at the level of power series generated uniform algebras. This is in view of the facts that (i) there exists an infinite dimensional uniform Banach algebra having a power series generator [1, Proposition 4]; and that (ii) a nuclear Banach space is finite dimensional (Dvoretzky-Rogers Theorem). In the following, the topological algebraic property of being a uniform algebra forces the linear 
topological property of nuclearity.

THEOREM 3.6. Let $A$ be a uniform Fréchet algebra with a power series generator. Suppose that $A$ is not a Banach algebra. Then $A$ is a nuclear space.

Proof: Let $\left(p_{k}\right)$ be as above. Then $p_{k}\left(f^{n}\right)=p_{k}(f)^{n}$ for all $k \in \mathbb{N}$ all $n \in \mathbb{Z}^{+}$ and for all $f \in A$. Let $x$ be a power series generator for $A$. Let $r_{k}=p_{k}(x)$. Then $\left(r_{k}\right)$ is an increasing sequence of non-negative numbers such that given $n \in \mathbb{N}$, there exists $k \in \mathbb{N}$ satisfying $r_{k}^{n}>0$. Now each $f \in A$ is of the form $f=\sum_{n=0}^{\infty} \lambda_{n} x^{n}$ with

$$
\sum_{n=0}^{\infty}\left|\lambda_{n}\right| r_{k}^{n}=\sum_{n=0}^{\infty}\left|\lambda_{n}\right| p_{k}(x)^{n}=\sum_{n=0}^{\infty}\left|\lambda_{n}\right| p_{k}\left(x^{n}\right)<\infty
$$

for each $k$. The functions $\omega_{k}(n)=r_{k}^{n}\left(n \in \mathbb{Z}^{+}\right)$satisfy $\omega_{k}(n+m)=\omega_{k}(n) \omega_{k}(m)$, so that $\ell^{1}\left(\mathbb{Z}^{+}, \omega\right)$ is a power series space $\wedge\left(\left\{\omega_{k}\right\}\right)$ in the sense of [10, Chapter 6]. The map

$$
\theta: \ell^{1}\left(\mathbb{Z}^{+}, \boldsymbol{\omega}\right) \rightarrow A, \theta\left(\sum_{n=0}^{\infty} \lambda_{n} X^{n}\right)=\sum_{n=0}^{\infty} \lambda_{n} x^{n}
$$

is a surjective homomorphism such that for each $k$,

$$
p_{k}\left(\sum_{n=0}^{\infty} \lambda_{n} x^{n}\right) \leqslant \sum_{n=0}^{\infty}\left|\lambda_{n}\right| \omega_{k}(n) .
$$

Thus $\theta$ is continuous; and $A \cong \ell^{1}\left(\mathbb{Z}^{+}, \omega\right) / \operatorname{ker} \theta$, a homeomorphic isomorphism in view of the Open Mapping Theorem. Since $\left(r_{k}\right)$ is an increasing sequence, it follows from the Grothendieck-Pietsch criterion ([10, Theorem 6.1.2, p. 98]) that $\ell^{1}\left(\mathbb{Z}^{+}, \omega\right)$ is nuclear. Since nuclearity is preserved under quotient ([10, Proposition 5.1.3, p. 86]), $A$ is nuclear. This completes the proof.

This recaptures the classical results that the algebras $H(U)$ and $\mathbb{E}$ are nuclear. More generally, it follows from [10, Theorem 6.1.3, p. 99] that a Beurling-Fréchet algebra $\ell\left(\mathbb{Z}^{+}, \omega\right)$ is nuclear if and only if $\ell^{1}\left(\mathbb{Z}^{+}, \omega\right)=\ell^{\infty}\left(\mathbb{Z}^{+}, \omega\right)$. Thus the algebra $A^{\infty}(\Gamma)$ is nuclear. Given weights $\boldsymbol{\omega}=\left(\omega_{k}\right), A(D, \omega)$ (and analogously $H^{\infty}(U, \boldsymbol{\omega})$ or $H(U, \boldsymbol{\omega})$ ) is nuclear if and only if for each $f \in A(D, \omega)$,

$$
\sup _{n}\left\{\frac{\left|f^{(n)}(0)\right|}{n !} \omega_{k}(n)\right\}<\infty
$$

for each $k$. The homomorphism $\theta$ of the above proof is used to prove the following supplements of Theorem 2.1 and [6, Chapter III, Section 7]. It also provides a Fréchet algebra analogue of $[\mathbf{1}$, Theorem 1]. We say that $x$ satisfies condition $(*)$ if

(i) $\exists M_{1}>0, M_{2}>0$ and for each $k$, there exists $m_{k} \in \mathbb{N}$ such that

$$
M_{1} n^{m_{k}} \leqslant p_{k}\left(x^{n}\right) \leqslant M_{2} n^{m_{k}} \forall n \in \mathbb{Z}^{+} \backslash\{0\}
$$


and

(ii) $\exists n_{0} \in \mathbb{N}$ such that $\left\{p_{k}\left(x^{n_{0}}\right): k \in \mathbb{N}\right\}$ is unbounded.

Theorem 3.7. Let $A$ be a Fréchet algebra having a power series generator $x$. The following hold

(1) Either $\operatorname{sp}(x)$ is totally disconnected or $A$ is isomorphic to $\ell^{\mathbf{1}}\left(\mathbb{Z}^{+}, \omega\right)$ for a sequence $\omega$ of weight functions on $\mathbb{Z}^{+}$.

(2) Suppose that $\operatorname{sp}(x)$ is not totally disconnected and $A$ is semisimple. Then the following hold.

(i) If $A$ is a $Q$-algebra, and if $x$ satisfies condition (*), then $A$ is isomorphic to $A^{\infty}(\Gamma)$.

(ii) If $A$ is not a $Q$-algebra, then $A$ is isomorphic to either $H(U)$ or $\mathbb{E}$.

Proof: In the notations of the proof of Theorem 3.6, let $\omega_{k}(n)=p_{k}\left(x^{n}\right)$, $r_{k}=\inf _{n \in \mathbb{Z}^{+}} \omega_{k}(n)^{1 / n}, r=\sup _{k \in \mathbb{N}} r_{k}$. Suppose there is

$$
f=\sum_{n=0}^{\infty} \lambda_{n} X^{n} \in \ell^{1}\left(\mathbb{Z}^{+}, \omega\right)
$$

such that

$$
\theta(f)=\sum_{n=0}^{\infty} \lambda_{n} x^{n}=0
$$

Then the power series $\sum_{n=0}^{\infty} \lambda_{n} z^{n}$ is absolutely convergent on every compact subset of $U_{r}=\{z \in \mathbb{C}:|z|<r\}^{n=0}$. Hence $f(z)=\sum_{n=0}^{\infty} \lambda_{n} z^{n}$ defines an analytic function on the region $U_{r}$, and $f(x)=0$. Thus sp $(x) \subset Z_{U_{r}}(f)$ the zero set of $f$ in $U_{r}$. Now $r=0$ if and only if $\operatorname{sp}(x)=\{0\}$ if and only if $A$ is local. Suppose $r \neq 0$. Now either $f=0$ or $Z_{U_{r}}(f)$ is a countable set consisting of isolated points, hence sp $(x)$ totally disconnected. Thus either $\operatorname{sp}(x)$ is totally disconnected, in which case $A \cong \ell^{1}\left(\mathbb{Z}^{+}, \omega\right) / \operatorname{ker} \theta$; or $\lambda_{n}=0$ for all $n$, so that $f=0$, and $\theta$ is one-one. In this case, $A \cong \ell^{1}\left(\mathbb{Z}^{+}, \boldsymbol{\omega}\right)$. Notice that $r>0$ if and only if $\ell^{1}\left(\mathbb{Z}^{+}, \omega\right)$ is semisimple. This proves (1).

Now assume that $\operatorname{sp}(x)$ is not totally disconncted and that $A$ is semisimple. Then the Gelfand space $M(A)$ is homeomorphic to $\mathrm{sp}(x)$. Now, for each $k, \overline{U_{r_{k}}} \subset \operatorname{sp}(x)$; hence $U_{r} \subset \operatorname{sp}(x)=\bigcup_{k=1}^{\infty} \bar{U}_{r_{k}} \subset \overline{U_{r}}$. Thus if $r \neq r_{k}$ for any $k$, then $\operatorname{sp}(x)=U_{r}$; if $r=r_{k}$ for some $k$, then $\operatorname{sp}(x)=\overline{U_{r}}$. Hence $\operatorname{sp}(x)=U_{r}$ or $\overline{U_{r}}$. Notice that by [11, Theorem 1] $\operatorname{sp}(x)$ is compact if and only if $A$ is a $Q$-algebra. As in the proof of 
Theorem 2.1, eithr $A \cong \mathcal{F}$; or each $\omega_{k}$ is a weight, so that $A \cong \bigcap_{k=1}^{\infty} \ell^{1}\left(\mathbb{Z}^{+}, \omega_{k}\right)$, hence

$$
M(A) \cong \bigcup_{k=1}^{\infty} M\left(\ell^{1}\left(\mathbb{Z}^{+}, \omega_{k}\right)\right) \cong \bigcup_{k=1}^{\infty} \bar{U}_{r_{k}} .
$$

Further by $[4$, p. 120$] \ell^{1}\left(\mathbb{Z}^{+}, \omega_{k}\right) \cong A\left(\bar{U}_{r_{k}}, \omega_{k}\right)$, where

$$
A\left(\bar{U}_{r_{k}}, \omega_{k}\right)=\left\{f \in C\left(\overline{U_{r_{k}}}\right): f \in H\left(U_{r_{k}}\right), \sum_{n=0}^{\infty}\left|\frac{f^{(n)}(0)}{n !}\right| \omega_{k}(n)<\infty\right\} .
$$

Indeed, the Gelfand transform

$$
y=\sum_{n=0}^{\infty} \lambda_{n} X^{n} \rightarrow \widehat{y}, \widehat{y}(z)=\sum_{n=0}^{\infty} \lambda_{n} z^{n}\left(z \in \overline{U_{r_{k}}}\right)
$$

establishes the isomorphim $\ell^{1}\left(\mathbb{Z}^{+}, \omega_{k}\right) \cong A\left(\bar{U}_{r_{k}}, \omega_{k}\right)$. Thus $A \cong \lim _{k} A\left(\bar{U}_{r_{k}} \omega_{k}\right)$. Suppose $A$ fails to be a $Q$-algebra. If $r=\infty$, then $A \cong \lim _{k} A\left(\bar{U}_{r_{k}}, \omega_{k}\right)=\mathbb{E}$. If $0<r<\infty$, then $A=\lim _{k} A\left(\bar{U}_{r_{k}}, \omega_{k}\right)=H\left(U_{r}\right)$ topologically as well. In view of the Riemann Mapping Theorem, $U_{r}$ can be replaced by $U_{1}$. This proves (ii). Finally to prove (i), assume that $A$ is a $Q$-algebra. Hence $M(A) \cong \overline{U_{r}} \cong \overline{U_{1}}$. By condition (*) (ii), there exists $n_{0} \in \mathbb{N}$ such that $\left\{p_{k}\left(x^{n_{0}}\right): k \in \mathbb{N}\right\}$ is unbounded; and by condition (*) (i), $m_{k} \rightarrow \infty$. Thus $y \in A$ if and only if the Gelfand transform $\widehat{y}=f$ for some function $f \in C(\Gamma)$ having Fourier series $f=\sum_{n \in \mathbb{Z}} \widehat{f}(n) e^{i n \theta}$ such that $\widehat{f}(n)=0$ for all $n \leqslant-1$ and

$$
\sum_{n=0}^{\infty}|\widehat{f}(n)| n^{m_{k}}<\infty
$$

for all $k \in \mathbb{N}$ by condition (*)(i). This happens if and only if $f \in A^{\infty}(\Gamma)$.

\section{REFERENCES}

[1] G.R. Allan, 'Commutative Banach algebras with a power series generator', in Radical Banach algebras and automatic continuity, (J.M. Bachar, W.G. Bade, P.C. Curtis, Jr., H.G. Dales and M.P. Thomas, Editors), Lecture Notes in Mathematics 975 (Springer-Verlag, Berlin. 1983), pp. 290-294.

[2] G.R. Allan, 'Fréchet algebras and formal power series', Studia Math. 119 (1996), 271-288.

[3] G. Carboni and A. Larotonda, 'An example of a Fréchet algebra which is a principle ideal domain', Studia Math. 138 (2000), 265-275.

[4] I.M. Gelfand, D. Raikov and G. Silov, Commutative normed rings (Chelsea Publ. Co., Bronx, New York, 1964). 
[5] H. Goldmann, Uniform Fréchet algebras, North Holland Mathematical Studies 162 (North Holland Publ. Co., Amsterdam, 1990).

[6] T. Husain, Orthogonal Schauder basis, Monographs and Textbooks in Pure and Applied Mathematics 134 (Marcel-Dekker Inc., New York, 1991).

[7] R.J. Loy, 'Continuity of derivations on topological algebras of power series', Bull. Austral. Math. Soc. 1 (1969), 419-424.

[8] R.J. Loy, 'Uniqueness of the Fréchet space topology on certain topological algebras', Bull. Austral. Math. Soc. 4 (1971), 1-7.

[9] R.J. Loy, 'Banach algebras of power series', J. Austral. Math. Soc. 17 (1974), 263-273.

[10] A. Pietsch, Nuclear locally convex spaces (Springer-Verlag, Berlin, Heidelberg, New York, 1972).

[11] W. Zelazko, 'On maximal ideals in commutative $m$-convex algebras', Studia Math. 58 (1976), 291-298.

\footnotetext{
Department of Mathematics

Sardar Patel University

Vallabh Vidyanagar 388120

Gujarat

India

e-mail: sjb@spu.ernet.in

coolpatel1@yahoo.com
} 